\title{
Uso de métodos não farmacológicos de alívio da dor durante o trabalho de parto e sua relação com a redução de ansiedade puerperal em uma maternidade da Rede SUS de Aracaju
}

\author{
Use of non-pharmacological methods of pain relief during labor and its relation to the reduction of \\ puerperal anxiety in a maternity hospital of the SUS Network of Aracaju \\ Utilización de métodos no farmacológicos de alivio del dolor durante el parto y su relación con la \\ reducción de la ansiedad puerperal en una maternidad de la Red SUS de Aracaju
}

Recebido: 10/04/2021 | Revisado: 16/04/2021 | Aceito: 20/04/2021 | Publicado: 25/04/2021

\author{
Luana Rocha de Souza \\ ORCID: https://orcid.org/0000-0002-5690-9323 \\ Universidade Tiradentes, Brasil \\ E-mail: luanapg.rocha28@gmail.com \\ Felipe Silveira de Faria \\ ORCID: https://orcid.org/0000-0001-5089-7168 \\ Universidade Tiradentes, Brasil \\ E-mail: felipesilveiradefaria@gmail.com \\ Larissa Wábia Santana de Almeida \\ ORCID: https://orcid.org/0000-0003-2598-1055 \\ Universidade Tiradentes, Brasil \\ E-mail: larissawabia@gmail.com \\ Letícia Andrade Santos \\ ORCID: https://orcid.org/0000-0002-5223-2781 \\ Universidade Tiradentes, Brasil \\ E-mail: leticia.asantos@souunit.com.br \\ Manuela Naiane Lima Barreto \\ ORCID: https://orcid.org/0000-0002-4487-9227 \\ Universidade Tiradentes, Brasil \\ E-mail: manuela.naiane@gmail.com \\ Débora Cristina Fontes Leite \\ ORCID: https://orcid.org/0000-0002-5396-2428 \\ Universidade Federal de Sergipe, Brasil \\ Universidade Tiradentes, Brasil \\ E-mail: deboraleite2006@hotmail.com
}

\begin{abstract}
Resumo
Este trabalho objetiva avaliar o impacto entre o uso de métodos não-farmacológicos de alívio da dor durante o parto e ansiedade puerperal na Maternidade de Risco Habitual, em Aracaju, verificando a frequência do uso destas técnicas e o nível de ansiedade das parturientes. Trata-se de estudo prospectivo, transversal, com abordagem quantitativa com 810 puérperas, de todas as idades, advindas dos 75 municípios do estado de Sergipe que buscaram a maternidade da Rede SUS de Aracaju. De set./2019 a fev./2020, os pesquisadores coletaram dados dos prontuários de puérperas nas primeiras 48 horas após o parto, mediante Termo de Consentimento Livre e Esclarecido (TCLE) ou Termo de Assentimento Livre e Esclarecido (TALE). Avaliou-se se as parturientes utilizaram métodos não farmacológicos de alívio da dor e foram aplicados os questionários IDATE-Traço e IDATE-Estado para aferição de ansiedade nas puérperas. Analisou-se os dados coletados estatisticamente pelo programa Jasp versão 0.12.1. O CEP da Universidade Tiradentes aprovou o trabalho (parecer: 3.695.763). O estudo demonstrou correlação positiva ( $\mathrm{p}$-value $<0,05)$ entre métodos não farmacológicos de alívio de dor e IDATE-TRAÇO (SCORE) e IDATE-ESTADO (SCORE), em que mulheres com maior score para ansiedade não usaram métodos não farmacológicos de alívio da dor. Conclui-se haver relação estatística significativa entre métodos não farmacológicos de alívio da dor e ansiedade puerperal.
\end{abstract}

Palavras-chave: Ansiedade; Trabalho de parto; Obstetrícia; Dor.

\begin{abstract}
This article aims to evaluate the statistical correlation between the use of non-pharmacological methods of pain relief during childbirth and puerperal anxiety at the Maternity, in Aracaju, verifying the frequency of use of these techniques and the level of anxiety of parturients. This is a prospective, cross-sectional study, with a quantitative approach with 810 puerperal women, of all ages, from the 75 municipalities in the state of Sergipe who sought the maternity of the
\end{abstract}


SUS Network of Aracaju. From Sep./2019 to Feb./2020, the researchers collected data from the medical records of postpartum women in the first 48 hours after giving birth, through Informed Consent Form (ICF) or Informed Consent Form (ICF). It was assessed whether the parturient women used non-pharmacological methods of pain relief and the IDATE-Trait and IDATE-State questionnaires were applied to measure anxiety in puerperae. The data collected were statistically analyzed by the program Jasp version 0.12.1. The CEP of Tiradentes University approved the study (opinion: 3.695.763). The study showed a positive relationship (p-value $<0.05$ ) between non-pharmacological methods of pain relief and IDATE-ANXiety (SCORE) and IDATE-STATE (SCORE), in the women with higher anxiety score without using the non-pharmacological methods of pain relief. Statistically based, we conclude that there is a significant relationship between non-pharmacological methods of pain relief and puerperal anxiety.

Keywords: Anxiety; Labour; Obstetrics; Pain.

\section{Resumen}

El objetivo de este trabajo es evaluar la correlación estadística entre el uso de métodos no farmacológicos de alivio del dolor durante el parto y la ansiedad puerperal en la Maternidad, en Aracaju, verificando la frecuencia de uso de estas técnicas y el nivel de ansiedad de las parturientas. Se trata de un estudio prospectivo, transversal, con abordaje cuantitativo con 810 puérperas, de todas las edades, provenientes de los 75 municipios del estado de Sergipe que buscan la maternidad de la Red SUS de Aracaju. Desde Sep./2019 hasta Feb./2020, los investigadores recogieron datos de las historias clínicas de las mujeres puérperas en las primeras 48 horas después del parto, a través de un Formulario de Consentimiento Libre e Informado (FICF) o de un Formulario de Consentimiento Informado (TALE). Se evaluó si las parturientas utilizaban métodos no farmacológicos para aliviar el dolor y se aplicaron los cuestionarios IDATE-Trait e IDATE-State para medir la ansiedad en las puérperas. Los datos recogidos fueron analizados estadísticamente por el programa Jasp versión 0.12.1. El CEP de la Universidad de Tiradentes aprobó el estudio (dictamen: 3.695.763). El estudio mostró una relación positiva (valor $\mathrm{p}<0,05$ ) entre los métodos no farmacológicos de alivio del dolor y el IDATE-ANXiety (SCORE) y el IDATE-SCORE, en el que las mujeres con mayor puntuación de ansiedad no utilizaban métodos no farmacológicos de alivio del dolor. Se concluye que existe una relación estadísticamente significativa entre los métodos no farmacológicos de alivio del dolor y la ansiedad puerperal.

Palabras clave: Ansiedad; Parto; Obstetricia; Dolor.

\section{Introdução}

A dor é parte integrante do trabalho de parto, sendo associada por muitos estudos à ansiedade durante este processo, de modo que, quando a dor é minimizada, a ansiedade também é aliviada (Almida et al, 2005). Essa dor resulta de complexas interações, de caráter inibitório e excitatório e, embora, seus mecanismos sejam semelhantes aos da dor aguda, existem fatores específicos do trabalho de parto de natureza neurofisiológica, obstétrica, psicológica e sociológica que interferem no seu limiar. Desta forma, uma vez que a dor é de caráter multidimensional, os analgésicos sozinhos não conseguem dirimi-la, de modo que as opções não farmacológicas podem auxiliar melhor a parturiente no alívio da dor, aumentando a tolerância à dor (Mafetoni \& Shimo, 2014), encorajando as parturientes, companheiros e familiares neste momento, e não associando a dor do parto ao medo, ao perigo e sofrimento. (Gayeski \& Brüggemann, 2010; Silva, Strapasson, \& Fischer, 2011; Almeida et al, 2005; Mafetoni \& Shimo, 2014).

Outrossim, quando os níveis de adrenalina estão altos, o sistema nervoso simpático é imediatamente ativado, aumentando os níveis plasmáticos do hormônio liberador de corticotrofinas, do hormônio adenocorticotrófico e do cortisol, elevando os níveis de estresse e ansiedade no trabalho de parto (Gayeski \& Brüggemann, 2010; Almeida et al, 2005). Tendo em vista esses aspectos, torna-se evidente que devem ser desenvolvidas ações para diminuir esses níveis. Neste contexto, recorre-se a métodos de alívio da dor, como os não-farmacológicos, que incluem a liberdade de adotar posturas e posições variadas, deambulação, respiração ritmada, comandos verbais e relaxamento, banhos de chuveiro e de imersão, toque e massagens e uso da bola (Silva, Strapasson, \& Fischer, 2011). Esses métodos baseiam-se em conhecimentos estruturados, mas que não necessitam de equipamentos sofisticados para sua utilização, podendo ser aplicados, até mesmo, pelo acompanhante de escolha da mulher (Gayeski \& Brüggemann, 2010).

A partir disso, este trabalho objetiva avaliar se há relação entre uso de métodos não-farmacológicos de alívio da dor durante o parto e ansiedade puerperal na Maternidade de Risco Habitual, em Aracaju/Sergipe. Além disso, tem por objetivos 
específicos verificar a frequência do uso de métodos não-farmacológicos de alívio da dor durante o trabalho de parto; avaliar o nível de ansiedade das parturientes; estudar a correlação estatística entre uso de métodos não farmacológicos de alívio da dor e ansiedade puerperal.

\section{Metodologia}

O presente estudo é um braço de uma pesquisa maior, que envolve os trabalhos "Avaliação dos componentes de ansiedade em pacientes no pós-parto imediato em uma maternidade da rede SUS em Aracaju/SE", de Almeida et al (2020) e "Impacto da sífilis congênita na ansiedade de pacientes no pós-parto imediato em uma maternidade da rede SUS de AracajuSE”, de Santos et al (2021), de modo que, na atual pesquisa, estudou-se as implicações da analgesia não farmacológica na ansiedade puerperal. Trata-se de um estudo transversal, de abordagem quantitativa, com amostra por conveniência, onde foram recrutadas para inclusão na pesquisa puérperas dos alojamentos conjuntos de uma maternidade filantrópica, de risco habitual na cidade de Aracaju, no estado de Sergipe, Nordeste brasileiro. É um estudo transversal, porque neste estudo observacional a situação de saúde de uma população é avaliada com base no estado de cada indivíduo que a compõe. Também é um estudo de prevalência, visto que mede a prevalência do uso de métodos não farmacológicos de alívio da dor em uma determinada população e em determinado momento (Estrela, 2018). Ademais, é um estudo de abordagem quantitativa, haja vista ser feita a coleta quantitativos ou numéricos por meio de medições de grandezas, em que são gerados conjuntos de dados que podem ser analisados através de técnicas matemáticas como a estatística (Pereira, 2018).

Esta maternidade, com 91\% dos leitos destinados ao SUS, realizou 10.920 partos em 2018, sendo a média mensal de 945,3 partos/mês. A prevalência de parto vaginal neste serviço em 2018 foi de 73\%. A população da amostra foi de puérperas provenientes dos 75 municípios do estado de Sergipe internadas na maternidade durante o período da coleta de dados, sendo o tamanho da amostra utilizou-se a fórmula de Barbetta (2010), considerando o número de partos realizados no serviço em 2018, com erro amostral de 5\%, o tamanho da amostra foi de 810 mulheres, dentre essas houve 7 perdas totais. No período de setembro de 2019 a fevereiro de 2020 os pesquisadores realizaram diariamente as entrevistas e a coleta dos dados das puérperas nas primeiras 48 horas após o parto.

Os critérios de inclusão foram: puérperas de todas as idades internadas na maternidade no período da coleta e que aceitem participar do estudo, assinando o Termo de Consentimento Livre e Esclarecido (TCLE) ou Termo de Assentimento Livre e Esclarecido (TALE). Foram excluídas da pesquisa pacientes que apresentarem história atual ou passada de depressão ou tratamento psiquiátrico, alcoolismo ou abuso de drogas, gestação gemelar, filhos estejam natimortos ou que sejam encaminhadas a Unidade de Terapias Intensiva. Dessa forma, foram avaliados alguns fatores clínicos e socioeconômicos como: idade, estado civil, se gravidez desejada/planejada, número de consultas pré-natal, tipo de parto, se gestação única ou múltipla, paridade com especificação do tipo de parto anterior, escolaridade da mãe, etnia, patologias gestacionais, opinião da paciente se o parto foi considerado "violento" e por quê, qualidade da acomodação hospitalar, qualidade da sala de parto, se utilizou algum método não farmacológico de alívio da dor, se foi incentivada a andar e se movimentar durante o parto, teve acompanhante de livre escolha, recebeu dieta líquida, realizou episiotomia, teve direcionamento dos puxos da dor durante o parto, qual a posição adotada no período expulsivo e realizou contato pele a pele com o recém-nascido. E dados em relação ao recém-nascido, como: apresentação do recém-nascido, sexo, idade gestacional, dados antropométricos, Apgar, realização de reanimação neonatal, presença de tocotrauma, se acompanha a genitora no Alojamento conjunto, amamentação na primeira hora pós-parto e dificuldade na amamentação (Almeida et al, 2020).

Em sequência foram aplicados o questionário IDATE-Traço e IDATE-Estado, instrumentos validados para aferição de ansiedade nas pacientes participantes do estudo (Delgado et al, 2016). Por fim, foi aplicado o questionário de qualidade de vida WHOQOL-bref que é um instrumento objetivo, recomendado pela OMS, que avalia "a percepção do indivíduo de sua 
posição no contexto da cultura e sistema de valores nos quais ele vive e em relação aos seus objetivos, expectativas, padrões e preocupações” (Almeida-Brasil et al, 2017).

À respeito dos riscos e benefícios, por se tratar de análise do prontuário, cartão da gestante e resposta ao questionário não apresentou riscos físicos, entretanto há riscos de constrangimento, quebra de sigilo e do anonimato. Para minimizar esses riscos os pesquisadores se comprometeram a respeitar a privacidade da paciente durante a coleta de dados, permitiram que ela somente responda o que se sentir confortável e guardaram as informações em local seguro e restrito. Além disso, somente os pesquisadores tiveram acesso a as informações que foram utilizadas exclusivamente para a realização deste estudo (Almeida et al, 2020).

Os dados coletados foram armazenados em um banco de dados no Microsoft Excel 2010 e analisados por intermédio de estatística pelo programa Jasp versão 0.12.1. Desse modo, todas as variáveis nominais do questionário sociodemográfico e clínico adotaram o número (1) para "Sim" e (2) para "Não" a fim de converter as palavras para números para serem lidas pelo programa (Almeida et al, 2020).

Para as análises estatísticas do questionário Idate foi necessário inverter na planilha as afirmações positivas; $1 ; 6 ; 7 ; 10 ; 13 ; 16$ e 19 no Idate-Traço e 1;2;5;8;10;11;15;16;19 e 20 no Idate-Estado, em seguida foi calculado o score e realizouse a correlação com os fatores clínicos e socioeconômicos e com WHOQOL-Bref (Almeida et al, 2020).

O WHOQOL-Bref é composto por 26 questões, duas de caráter geral sobre qualidade de vida e saúde e 24 questões ou facetas que foram divididas em quatro domínios cujas características estão apresentadas a seguir. O domínio Físico representa a percepção do indivíduo sobre sua condição física. O domínio Psicológico é a percepção do indivíduo sobre sua condição afetiva e cognitiva, ou seja, representa o grau de sua satisfação com a vida. Já o domínio Relações Sociais caracteriza a percepção do indivíduo sobre os relacionamentos sociais e os papéis sociais adotados na vida. Por fim, o domínio Meio Ambiente retrata a percepção do indivíduo sobre os aspectos diversos relacionados ao ambiente onde vive, levando em conta a infraestrutura desse ambiente (Nunes \& Freire, 2020).

Os resultados do WHOQOL-Bref foram transformados da seguinte forma: o primeiro passo foi estabelecer o valor de cada alternativa escolhida nas questões. Todas as questões, com exceção de três (Q3, Q4 e Q26), são respondidas em direção positiva, ou seja, quanto maior o número escolhido para a resposta, maior será o seu valor. As questões Q3, Q4 e Q26 foram formuladas na direção negativa, logo sua pontuação é invertida: (1=5), (2=4), (3=3), (4=2) e (5=1) (Amaral, 2015).

$\mathrm{O}$ instrumento não admite um escore total de $\mathrm{QV}$, considerando a premissa de que QV é um construto multidimensional; portanto cada domínio é pontuado de forma independente. A determinação dos escores dos domínios é realizada multiplicando-se a média de todos os itens incluídos dentro de um domínio por quatro. Dessa forma, o escore pode variar de zero a cem, sendo que quanto maior o valor, melhor é o domínio de qualidade de vida (Nunes \& Freire, 2020). A avaliação foi realizada com peso conforme preconiza o WHOQOL-Bref. É preciso ressaltar que este questionário é bem aceito e válido na população de puérperas e pode ser usado em contextos clínicos pós-natais ou para avaliar os efeitos da intervenção em pesquisas (Amaral, 2015).

A correlação entre os domínios de Qualidade de Vida e os níveis ansiedade foram realizadas através do teste ANOVA duas vias seguido do pós-teste de Tukey. O nível de significância adotado para todas as análises foi de 5\%. Este trabalho foi aprovado no CEP da Universidade Tiradentes sob o parecer 3.695.763. Todas as pacientes assinaram o Termo de Consentimento Livre e Esclarecido ou o Termo de Assentimento Livre Esclarecido (Almeida et al, 2020).

\section{Resultados}

A amostra do estudo totalizou 810 mulheres, com idade média de 25,5 anos, pardas em sua maioria (66.54\%) e estado civil solteira correspondendo a $48.39 \%$ delas. Em relação ao perfil socioeconômico das puérperas, 43,82\% não 
concluíram o ensino fundamental e cerca de 5,56\% nunca estudaram. 49,87\% das mulheres responderam como sendo donas de casa, $55,44 \%$ afirmaram residir em municípios do interior de Sergipe e 40,62\% na capital. Ademais, $62,46 \%$ delas responderam não trabalhar atualmente, de modo que a renda da casa para 59,38\% é de até 1 salário mínimo. O número médio de pessoas na residência (incluindo o recém-nascido) é de 4,483, sendo que 91,61\% residem em casas.

O presente estudo demonstrou relação positiva entre métodos não farmacológicos de alívio de dor e IDATE-TRAÇO (SCORE), em que mulheres com maior score para ansiedade necessitariam de uso de métodos não farmacológicos de alívio da dor e as que os utilizaram tiveram impacto negativo na pontuação IDATE - TRAÇO de ansiedade (p-value < 0,05). Entretanto, observou-se que menos da metade das participantes do estudo, apenas $22.716 \%$, usaram as técnicas não farmacológicas de alívio da dor. Esta relação estatística relevante deve-se, provavelmente, à disponibilidade heterogênea destas práticas no hospital, em que, embora sejam práticas relevantes para o alívio de ansiedade nas parturientes, não estão amplamente distribuídas no serviço.

Sobre a relação entre o uso de métodos não farmacológicos de alívio da dor e o IDATE TRAÇO (SCORE), os resultados obtidos pelo presente estudo são mostrados na Tabela 1, em que se deve observar a relação estatística significativa e positiva entre usar o método não farmacológico para aliviar a dor e o IDATE TRAÇO (SCORE).

Tabela 1: Relação estatística entre uso de métodos não farmacológicos de alívio da dor e IDATE - TRAÇO (SCORE)

\begin{tabular}{|c|c|c|c|c|}
\hline Variável & & IDATE TRAÇO (SCORE) & $\begin{array}{l}\text { Método não farmacológico } \\
\text { de alívio da dor }\end{array}$ & $\begin{array}{c}\text { Incentivo a andar e se } \\
\text { movimentar }\end{array}$ \\
\hline \multirow[t]{4}{*}{$\begin{array}{l}\text { 1. IDATE TRAÇO } \\
\text { (SCORE) }\end{array}$} & Pearson's r & - & - & - \\
\hline & p-value & - & - & - \\
\hline & Upper 95\% CI & - & - & - \\
\hline & Lower $95 \% \mathrm{CI}$ & - & $\ldots$ & 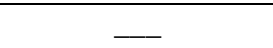 \\
\hline \multirow{4}{*}{$\begin{array}{l}\text { 2. Método não } \\
\text { farmacológico de alivio } \\
\text { de dor }\end{array}$} & Pearson's $r$ & $0.074 *$ & - & - \\
\hline & p-value & 0.036 & - & - \\
\hline & Upper 95\% CI & 0.143 & - & - \\
\hline & Lower $95 \% \mathrm{CI}$ & 0.005 & $\ldots$ & - \\
\hline \multirow{4}{*}{$\begin{array}{l}\text { 3. Incentivo a andar e se } \\
\text { movimentar }\end{array}$} & Pearson's $r$ & 0.037 & $0.391 * * *$ & - \\
\hline & p-value & 0.293 & $<.001$ & - \\
\hline & Upper 95\% CI & 0.107 & 0.448 & - \\
\hline & Lower 95\% CI & -0.032 & 0.330 & - \\
\hline
\end{tabular}

$* \mathrm{p}<.05, * * \mathrm{p}<.01, * * * \mathrm{p}<.001$.

Fonte: Dados da pesquisa.

Além disso, houve correlação estatística relevante entre usar ou não Método não farmacológico de alivio de dor e IDATE ESTADO (SCORE), em que o não uso de Métodos não farmacológicos de alivio da dor impactaria na presença de ansiedade puerperal, permitindo que os níveis de ansiedade ficassem elevados ( $\mathrm{p}$-value $<0,05$ ). Isto pode se dever ao fato de que a presença de dor pode causar ansiedade. 
Sobre a relação entre o uso de métodos não farmacológicos de alívio da dor e o IDATE ESTADO (SCORE), os resultados obtidos pelo presente estudo são mostrados na Tabela 2, em que se deve observar a relação estatística significativa e positiva entre usar o método não farmacológico para aliviar a dor e o IDATE ESTADO (SCORE), isto é, entre a ansiedade. Ademais, deve-se perceber pela mesma tabela que há uma relação estatística relevante entre o incentivo a andar e se movimentar e os métodos não farmacológicos de alívio da dor ( $\mathrm{p}$-value < 0.001 ), evidenciando o incentivo a andar e se movimentar como importante método não farmacológico de alívio de dor, o qual, inclusive, é um dos mais utilizados na Maternidade avaliada pelo presente estudo.

Tabela 2: Relação entre uso de métodos não farmacológicos de alívio da dor e IDATE ESTADO (SCORE).

\begin{tabular}{|c|c|c|c|c|}
\hline Variável & & $\begin{array}{c}\text { IDATE ESTADO } \\
\text { (SCORE) }\end{array}$ & $\begin{array}{c}\text { Método não } \\
\text { farmacológico de alívio } \\
\text { da dor }\end{array}$ & $\begin{array}{c}\text { Incentivo a andar e se } \\
\text { movimentar }\end{array}$ \\
\hline \multirow[t]{4}{*}{$\begin{array}{l}\text { 1. IDATE ESTADO } \\
\text { (SCORE) }\end{array}$} & Pearson's r & - & $\ldots$ & - \\
\hline & $\mathrm{p}$-value & - & - & - \\
\hline & Upper 95\% CI & - & $\ldots$ & - \\
\hline & Lower $95 \%$ CI & - & $\ldots$ & - \\
\hline \multirow{4}{*}{$\begin{array}{l}\text { 2. Método não } \\
\text { farmacológico de alívio } \\
\text { da dor }\end{array}$} & Pearson's $r$ & $0.079 *$ & $\ldots$ & \\
\hline & p-value & 0.027 & $\ldots$ & \\
\hline & Upper 95\% CI & 0.147 & $\ldots$ & \\
\hline & Lower 95\% CI & 0.009 & $\ldots$ & \\
\hline \multirow[t]{4}{*}{$\begin{array}{l}\text { 3. Incentivo a andar e se } \\
\text { movimentar }\end{array}$} & Pearson's $r$ & 0.035 & $0.391 * * *$ & - \\
\hline & p-value & 0.321 & $<.001$ & - \\
\hline & Upper 95\% CI & 0.105 & 0.448 & - \\
\hline & Lower 95\% CI & -0.034 & 0.330 & - \\
\hline
\end{tabular}

$* \mathrm{p}<.05, * * \mathrm{p}<.01, * * * \mathrm{p}<.001$

Fonte: Dados da pesquisa.

Houve ainda correlação estatística relevante entre ter ou não Acompanhante de livre escolha e IDATE ESTADO (SCORE), em que não ter acompanhante de livre escolha causaria ansiedade puerperal, bem como a presença do acompanhante atenuaria a tensão e ansiedade desta mulher durante o trabalho de parto (p-value $<0,01$ ). Isto pode se dever ao fato de que, sem um acompanhante, essa mulher pode ter se sentido sem apoio emocional durante o parto.

Na Tabela 3 observa-se a relação entre a presença do acompanhante de livre escolha e o IDATE ESTADO (SCORE), em que a presença do acompanhante de livre escolha teria impacto positivo sobre a ansiedade, reduzindo-a. 
Tabela 3: Relação entre Acompanhante de livre escolha e o IDATE ESTADO (SCORE).

\begin{tabular}{|l|l|c|}
\hline \multicolumn{1}{|c|}{ Variável } & & IDATE ESTADO (SCORE) \\
\hline 1. IDATE ESTADO (SCORE) & Pearson's r & - \\
\hline & p-value & - \\
\hline & Upper 95\% CI & $0.092^{* *}$ \\
\hline 2. Acompanhante de livre escolha & Lower 95\% CI & 0.010 \\
\hline & Pearson's r & 0.160 \\
\hline & p-value & 0.022 \\
\hline
\end{tabular}

$* \mathrm{p}<.05, * * \mathrm{p}<.01, * * * \mathrm{p}<.001$.

Fonte: Dados da pesquisa.

Entre as 810 gestantes entrevistadas, $22.716 \%$ usaram método não farmacológico de alívio da dor, sendo que, destas, apenas $46,67 \%$ foi incentivada a deambular. $95.185 \%$ do total da amostra tiveram acompanhante de livre escolha. E $64.321 \%$ do total das mulheres tiveram o parto em posição ginecológica, sendo que a posição ginecológica foi um fator de aumento no score da ansiedade (p-value < 0,05), sugerindo que esta não é a posição de parto mais confortável para a mãe, haja vista também existirem outras.

Na Tabela 4 é possível visualizar a porcentagem de mulheres que utilizaram métodos não farmacológicos de alívio da dor, como a deambulação, bem como as que tiveram condições de parto mais favoráveis para redução da ansiedade, como posição não litotômica e acompanhante de livre escolha.

Tabela 4: Dados da sala de parto.

\begin{tabular}{|c|c|}
\hline & SIM \\
\hline Uso de métodos não farmacológicos de alívio da dor & $22.716 \%$ \\
\hline Incentivo à deambulação & $10,62 \%$ \\
\hline Parto em posição ginecológica & $64.321 \%$ \\
\hline Acompanhante de livre escolha & $95.185 \%$ \\
\hline
\end{tabular}

Fonte: Dados da pesquisa.

\section{Discussão}

\section{Uso de métodos não farmacológicos de alívio da dor e redução da ansiedade pós-parto}

O presente estudo evidenciou que o não uso de Métodos não farmacológicos de alivio da dor impacta na presença de ansiedade puerperal, permitindo que os níveis de ansiedade ficassem elevados. Isto pode se dever ao fato de que a presença de dor e o não alívio desta podem causar ansiedade (Almeida et al, 2005). Corroborando os resultados do presente trabalho, a literatura demonstra que o uso de métodos não farmacológicos de alívio da dor impacta positivamente na redução da ansiedade puerperal, haja vista estas técnicas promoverem relaxamento, calmaria, diminuição da dor e alívio da ansiedade (Mascarenhas et al, 2019; Almeida et al, 2021). Outrossim, quando a mãe já possui traços de personalidade com componente de ansiedade, esta, durante o trabalho de parto, pode aumentar a percepção de dor oriundas das contrações, razão pela qual as práticas não farmacológicas devem ser utilizadas para reduzir a dor e a ansiedade associada. Conforme a literatura, os métodos não farmacológicos de alívio da dor mais utilizados nas maternidades brasileira pelas puérperas e mais conhecidos por proporcionarem este conforto são os banhos de aspersão, a deambulação, mudanças de posições (cócoras, sentada, 
agachamento) durante o trabalho de parto, técnicas de controle da respiração, massagens e bola suíça. Estes métodos também dialogam com as Práticas Integrativas e Complementares em Saúde, as PICS, as quais já vêm sendo realizadas em saúde da mulher nas diversas fases da vida, incluindo período gestacional, parturição e puerpério, e impactando positivamente para alívio do estresse e ansiedade (Souza et al, 2020). Ademais, a presença do acompanhante e as orientações e apoio recebidos dos profissionais também proporcionam conforto, apoio, força, tranquilidade, confiança, redução da insegurança, do medo e da própria ansiedade, ratificando os resultados do presente trabalho (Dias et al., 2018).

Dentre os métodos não farmacológicos, o banho de chuveiro é apresentado na literatura como um dos que, não apenas aliviam a dor, como também reduzem a pressão arterial, promovem o aumento da dilatação do colo uterino e, além disso, é uma medida de baixo custo e fácil de ser empregada (Dias et al., 2018). Em estudo realizado por Gayeski e Brüggemann (2010), a aromaterapia com essências diluídas na água associada a massagens também foi apontada como método não farmacológico de alívio da dor capaz de reduzir a dor, a ansiedade e o medo em $86 \%$ das mulheres que receberam a intervenção. Estes estudos mostram-se de acordo com os achados do presente trabalho, confirmando a eficácia de métodos não farmacológicos para alívio da ansiedade puerperal.

Embora os métodos não farmacológicos de alívio da dor sejam bastante positivos para a saúde mental da mãe durante o trabalho de parto, no estudo de Hanum et al (2017), que avaliou a percepção das parturientes de uma maternidade pública do estado de Goiás sobre o uso dos métodos não farmacológicos de alívio da dor durante o trabalho de parto, os resultados revelaram que 73,8\% não foram informadas sobre a existências de métodos não farmacológicos de alívio da dor. Este dado, inclusive, ratifica os dados encontrados pelo presente trabalho, em que apenas $22.716 \%$ usaram método não farmacológico de alívio da dor, sendo que, destas, apenas $46,67 \%$ foram incentivadas a se movimentar, ou seja, mais da metade das mulheres estudadas não se movimentou durante o trabalho de parto, apesar da deambulação e as posições verticais reduzirem a dor e a duração desse período. Já no estudo de Monguilhott et al. (2020), em uma análise da assistência às parturientes em serviços públicos da região Sul, a maioria das puérperas foi incentivada a movimentar-se $(59,2 \%)$, mas poucas fizeram uso de algum método não farmacológico para alívio da dor (32,7\%), o que se aproxima também dos achados do presente estudo.

Outro trabalho que avaliou o perfil da assistência obstétrica em uma maternidade da Rede SUS no estado do Piaú evidenciou que a maioria das mulheres $(63,8 \%)$ usou métodos não farmacológicos para alívio da dor, sugerindo uma heterogeneidade entre os diversos estados brasileiros a respeito desta assistência (Resende, Lopes, \& Bonfim, 2020).

\section{Posição do trabalho de parto}

No presente estudo, dentre as 810 mulheres da amostra, a maioria delas, $64.321 \%$, teve o parto em posição ginecológica, não adotando outras posições mais confortáveis para alívio da dor. Este dado encontra-se em consonância com a literatura, a qual evidencia que a posição de litotomia tem sido uma regra ao longo dos anos, alcançando mais de $90 \%$ das parturientes de risco habitual (Leal et al, 2014), e visto que mais da metade dos partos realizados em uma maternidade da Rede SUS do Piauí também ocorreram em posição litotômica (60,3\%) (Resende, Lopes, \& Bonfim, 2020). Outrossim, o estudo de Monguilhott et al. (2020) descreve que, durante o parto, pouquíssimas das mulheres avaliadas puderam escolher posição diferente da litotômica para parir $(5,1 \%)$, apesar dos benefícios das posições verticalizadas para a mulher e para o feto, por serem mais confortáveis, reduzindo a dor, a duração deste período e, consequentemente, a ansiedade.

Estes dados sugerem que, de uma forma geral, o potencial terapêutico dos métodos não farmacológicos de alívio da dor ainda é pouco aproveitado nas maternidades brasileiras da Rede SUS e até desconhecido (Sousa, Sena, \& Cunha, 2021; Alves et al, 2021; Santana et al, 2020), e que a execução dessas práticas ainda é muito pontual ao longo do país e está muito relacionada à filosofia assistencial do serviço (Monguilhott et al, 2020). Sugere-se ainda que há uma falta de interesse de 
profissionais e gestores de serviços de saúde na produção da atenção obstétrica e neonatal de melhor qualidade, dificultando a consolidação do uso dos diversos métodos não farmacológicos de alívio da dor como parte da rotina obstétrica.

\section{Presença do acompanhante durante o trabalho de parto}

O presente estudo evidenciou que não ter acompanhante de livre escolha causaria ansiedade puerperal, bem como a presença do acompanhante atenuaria a tensão e ansiedade desta mulher durante o trabalho de parto. Isto pode se dever ao fato de que, as puérperas do estudo que estavam sem um acompanhante de sua escolha possam ter se sentido sem apoio emocional suficiente durante o parto (Dias et al., 2018).

Ainda no estudo de Hanum et al (2017), um total de $99 \%$ revelou o conhecimento da presença do acompanhante na unidade. No estudo de Monguilhott et al. (2020), por sua vez, 51,7\% das entrevistadas permaneceu com o acompanhante durante o trabalho de parto. Entretanto, menos da metade teve a presença do acompanhante durante o parto (39,4\%). Estes dois estudos mostram-se contrastantes entre si em relação a esses dados, porém os resultados de Hanum et al (2017) não diferem muito do presente estudo, o qual demonstrou que $95.185 \%$ das parturientes tiveram acompanhante de livre escolha.

Esses achados podem estar relacionados à filosofia assistencial implementada nas instituições de saúde, uma vez que, ao permitir o acompanhante, outras práticas também são reconhecidas como benéficas e passam a fazer parte da rotina, conforme já mencionado em outras pesquisas que retratam experiências locais no Brasil. Além disso, quando o acompanhante é acolhido e estimulado a desenvolver ações de conforto físico, ele participa da aplicação de métodos não farmacológicos de alívio da dor (Monguilhott et al, 2020), de modo que a sua presença em sala impacta positivamente na redução da ansiedade puerperal.

Desta forma, os resultados reforçam a importância de investimentos nos hospitais maternidade e casas de parto para a inserção de programas e protocolos de incentivo ao uso dos métodos não farmacológicos no trabalho de parto, não apenas para que seja prestada uma assistência humanizada, mas também pelo fato de que as práticas não farmacológicas de alívio da dor atenuam a ansiedade, reduzindo, inclusive, a percepção de dor da mãe durante as contrações. Sugere-se ainda que estudos sejam realizados para verificar a disseminação de informações sobre estes métodos, ainda no pré-natal e o conhecimento dos profissionais de saúde sobre eles. Espera-se que esse estudo conscientize profissionais e as mulheres sobre a utilização dos métodos não farmacológicos (Mielke, Gouveia, \& Gonçalves, 2019) e que as instituições busquem a implementação desses para desmitificar questões relacionadas ao parto normal (Dias et al., 2018).

\section{Conclusão}

Conclui-se haver relação estatística significativa entre métodos não farmacológicos de alívio da dor e ansiedade puerperal, de modo que o uso desses métodos é capaz de reduzir a ansiedade puerperal. Ademais, o presente estudo revela correlação estatística positiva entre a presença do acompanhante de livre-escolha e a ansiedade puerperal, sugerindo, com base na literatura, que a presença do acompanhante também seria fundamental na diminuição dos níveis de ansiedade puerperal. Os resultados sugerem ainda que o uso de métodos não farmacológicos de alívio da dor nesta maternidade se Aracaju da Rede SUS ainda não é amplo o suficiente, dado que se apoia na literatura como parte de uma realidade nacional. Outrossim, o estudo aponta a necessidade de uso de métodos não farmacológicos de alívio da dor pelo fato de que a própria a ansiedade pode aumentar a percepção de dor durante o trabalho de parto, de modo que mães que já possuem traços para ansiedade podem se sentir mais desconfortáveis durante a parturição.

O presente trabalho ainda abre portas para outras investigações sobre a temática em questão através de estudos em que o número de participantes seja maior, sendo relevante que se faça comparações entre a aplicação de métodos não farmacológicos de alívio da dor durante a parturição e o nível socioeconômico das pacientes, avaliando-se melhor este uso em 
mulheres de condições socioeconômicas distintas. Além disso, seria relevante avaliar por meio de pesquisas maiores os métodos não farmacológicos de alívio da dor mais empregados nas maternidades rede SUS, bem como avaliar quais posições ginecológicas impactariam mais no alívio da dor e ansiedade puerperal, já que este estudo traz dados apenas sobre o impacto negativo da posição litotômica no parto.

\section{Referências}

Almeida-Brasil, C. C., Silveira, M. R., Silva, K. R., Lima, M. G., Faria, C. D. C. M., Cardoso, C. L., Menzel, H. J. K., \& Ceccato, M. G. B. (2017). Qualidade de vida e características associadas: aplicação do WHOQOL-BREF no contexto da Atenção Primária à Saúde. Ciência \& Saúde Coletiva, $22(5), 1705-1716$.

Almeida, C. F. S., Graepp-Fontoura, I., Tavares, H. S. A., Lima, L. N. F., Santos, F. S., Neto, M. S., Fonseca, S. C. T., Ribeiro, R. S., Araújo, S. M. N., Feitosa, M. O., Santos, R. M. M. S, Lima, C. C. D., Almeida, V. F. M., Holanda, N. B. A., Ribeiro, S. B., Silva, L. P., \& Fontoura, V. M. (2019). Métodos alternativos para alívio da dor no parto normal: revisão sistemática. Research, Society and Development, 10(1).

Almeida, L. W. S., Santos, L. A., Faria, F. S., Souza, L. R., Barreto, M. N. L., \& Leite, D. C. F. (2020). Avaliação dos componentes de ansiedade em pacientes no pós-parto imediato em uma maternidade da rede SUS em Aracaju/SE. Brazilian Journal of Health Review, 3(6).

Almeida, N. A. M., Souza, J. T., Bachion, M. M., \& Silveira, N. A. (2005). Utilização de técnicas de respiração e relaxamento para alívio de dor e ansiedade no processo de parturição. Rev Latino-am Enfermagem, 13(1), 52-58.

Alves, A. P. C., Alves, A. S., Tamboril, T. M., Menezes, V. B. B., Barros, L. O., Medeiros, R. F. B., Carvalho, C. G. M., \& Linard, C. F. B. M. (2021). Perfil e percepção das puérperas em relação ao trabalho de parto humanizado. Brazilian Applied Science Review, 5(1), $584-603$.

Amaral, R. C. (2015). Avaliação da qualidade de vida de pacientes adultos com distúrbio do desenvolvimento sexual (DDS) 46, XX e 46, XY em uma larga coorte de um único centro terciário (Tese de Doutorado em Ciências Médicas, Faculdade de Medicina da Universidade de São Paulo). Retirado de https://teses.usp.br/teses/disponiveis/5/5166/tde-05102015-090623/publico/RitadeCassiadoAmaralVersaoCorrigida.pdf.

Delgado, A. M., Freire, A. B., Wanderley, E. L. S., \& Lemos, A. (2016). Análise da validade de constructo e consistência interna da escala de estado de ansiedade (E-Ansiedade) do inventário de ansiedade traço-estado (Idate) para gestantes em trabalho de parto. Rev Bras Ginecol Obstet, 38 (11), 531-537.

Dias, E. G., Ferreira, A. R. M., Martins, A. M. C., Jesus, M. M., \& Alves, J. C. S. (2018). Eficiência de métodos não farmacológicos para alívio da dor no trabalho de parto normal. Enferm. Foco, 9(2), 35-39.

Estrela, C. (2018). Metodologia científica: ciência, ensino, pesquisa. Artes Médicas.

Gayeski, M. E., Brüggemann, O. M. (2010). Métodos não farmacológicos para alívio da dor no trabalho de parto: uma revisão sistemática. Texto Contexto Enferm, 19(4), 774-782.

Hanum, S. P., Mattos, D. V., Matão, M. E. L., \& Martins, C. A. (2017). Estratégias não farmacológicas para o alívio da dor no trabalho de parto: efetividade sob a ótica da parturiente. Rev enferm UFPE on line, 11(8), 3303-3309.

Leal, M. C., Pereira, A. P. E., Domingues, R. M. S. M., Filha, M. M. T., Dias, M. A. B., Nakamura-Pereira, M., Bastos, M. H., \& Gama, S. G. N. (2014). Intervenções obstétricas durante o trabalho de parto e parto em mulheres brasileiras de risco habitual. Cad. Saúde Pública, 30, 17-47.

Mafetoni, R. R., \& Shimo, A. K. K. (2014). Métodos não farmacológicos para alívio da dor no trabalho de parto: revisão integrativa. Rev Min Enferm., 18(2), 505-512.

Mascarenhas, V. H., Lima, T. R., Silva, F. M., Negreiros, F. S., Santos, J. D., Moura, M. A., Gouveia, M. T. O., \& Furtado Jorge, H. M. (2019). Evidências científicas sobre métodos não farmacológicos para alívio a dor do parto. Acta Paul Enferm, 32(3), 350-7.

Mielke, K. C., Gouveia, H. G., \& Gonçalves, A. C. (2019). A prática de métodos não farmacológicos para o alívio da dor de parto em um hospital universitário no Brasil. Av Enferm, 37(1), 47-55.

Monguilhott, J. J. C., Brüggemann, O. M., Freitas, P. F., \& D’Orsi, E. (2018). Nascer no Brasil: a presença do acompanhante favorece a aplicação das boas práticas na atenção ao parto na região Sul. Rev Saude Publica, 52(1), 1-11.

Nunes, M. F., \& Freire, M. C. M. (2006). Qualidade de vida de cirurgiões-dentistas que atuam em serviço público. Rev Saúde Pública, 40(6), 1019-1026.

Pereira, A. S. (2018). Metodologia da pesquisa científica https://repositorio.ufsm.br/bitstream/handle/1/15824/Lic_Computacao_Metodologia-PesquisaCientifica.pdf?sequence $=1$

Resende, M. T. S., Lopes, D. S., \& Bonfim, E. G. (2020). Perfil da assistência ao parto em uma maternidade pública. Rev. Bras. Saúde Mater. Infant., 20(3), 871-878.

Santana, C. S., Oliveira, A. M. N., Medeiros, S. P., Cardoso, V. M., Silva, M. R. S., \& Cezar-Vaz, M. R. (2020). Expectativas e sentimentos das puérperas acerca do trabalho de parto e parto. Research, Society and Development, 9(9), 1-18.

Santos, L. A. (2021). Impacto da sífilis congênita na ansiedade de pacientes no pós-parto imediato em uma maternidade da rede SUS de Aracaju-SE. In A. (Ed.), Práticas Preventivas e Práticas Curativas na Medicina 2 (pp. 44-50). Ponta Grossa: Atena Editora.

Silva, E. F., Strapasson, M. R., \& Fischer, A. C. S. (2011). Métodos não farmacológicos de alívio da dor durante trabalho de parto e parto. R. Enferm. UFSM, $1(2), 261-271$. 
Research, Society and Development, v. 10, n. 5, e21410514899, 2021

(CC BY 4.0) | ISSN 2525-3409 | DOI: http://dx.doi.org/10.33448/rsd-v10i5.14899

Sousa, K. H. J. F., Sena, A. F., \& Cunha, K. J. B. (2021). Tecnologias não farmacológicas e tempo de trabalho de parto e parto: Revisão sistemática sem metanálise. Rev Enferm Atual In Derme, 95(33).

Souza, V. A., Machado, G. N., Arrué, A. M., Luzardo, A. R., Jantsch, L. B., \& Danski, M. T. R. (2020). As Práticas Integrativas e Complementares na atenção à saúde da mulher. Research, Society and Development, 9(8), 1-23.

Webster, J., Nicholas, C., Velacott, C., Cridland, N., \& Fawcett, L. (2010). Validation of the WHOQOL-BREF among women fallowing childbirth. Aust New Zeal J Obstet Gynaecol, 50(2), 132-137.

Zanei, S. S. V. (2006). Análise dos instrumentos de avaliação de qualidade de vida WHOQOL-bref e SF-36: confiabilidade, validade e concordância entre pacientes de Unidades de Terapia Intensiva e seus familiares. (Tese de Doutorado em Enfermagem na Saúde do Adulto, Escola de Enfermagem da Universidade de São Paulo). https://www.teses.usp.br/teses/disponiveis/7/7139/tde-21032006-154203/publico/06_TeseZaneiSSV_EEUSP.pdf 